\title{
Toward Active Tremor Canceling in Handheld Microsurgical Instruments
}

\author{
Cameron N. Riviere, Member, IEEE, Wei Tech Ang, Student Member, IEEE, and Pradeep K. Khosla, Fellow, IEEE
}

\begin{abstract}
This paper describes research in active instruments for enhanced accuracy in microsurgery. The aim is to make accuracy enhancement as transparent to the surgeon as possible. Rather than using a robotic arm, we have taken the novel approach of developing a handheld instrument that senses its own movement, distinguishes between desired and undesired motion, and deflects its tip to perform active compensation of the undesired component. The research has therefore required work in quantification and modeling of instrument motion, filtering algorithms for tremor and other erroneous movements, and development of handheld electromechanical systems to perform active error compensation. The paper introduces the systems developed in this research and presents preliminary results.
\end{abstract}

Index Terms-Adaptive noise canceling, human performance augmentation, medical robotics, microsurgery, tremor.

\section{INTRODUCTION}

I NACCURACY in microsurgery has long been a concern [1]. Limitations on human accuracy in manual manipulation restrict the types of microsurgical procedures that are feasible, and hinder the quality of those that are performed. Effective suppression of involuntary or undesired motion in microsurgery, therefore, would not only improve accuracy in existing procedures, but would also likely open the way to new types of procedures. One example is retinal vein cannulation for injection of anticoagulants to treat occlusions. This procedure holds the potential of curing a major cause of blindness, estimated to affect $1.6 \%$ of persons aged 49 years or older [2], but is generally considered infeasible with existing manual techniques due to the imprecision of the surgeon [3]. Another ophthalmologic example is arteriovenous sheathotomy, which has engendered recent interest as a possible treatment for

Manuscript received June 14, 2002; revised March 14, 2003. This paper was recommended for publication by Associate Editor P. Dario and Editor R. Taylor upon evaluation of the reviewers' comments. This work was supported in part by the National Institutes of Health under Grant 1 R01 EB000526 and Grant 1 R21 RR13383, in part by the R. Green Annan Fund and the Lettie B. Trognitz Fund of The Pittsburgh Foundation, in part by the National Science Foundation under Grant EEC-9731748, and in part by the Focused Giving Program of Johnson \& Johnson. This paper was presented in part at the 23rd Annual IEEE Engineering in Medicine and Biology Society International Conference, Istanbul, Turkey, October, 2001; in part at the IEEE/RSJ International Conference on Intelligent Robots and Systems, Maui, HI, October 29-November 3, 2001; and in part at the 28th Annual IEEE Northeast Bioengineering Conference, Philadelphia, PA, April, 2002

C. N. Riviere and W. T. Ang are with the Robotics Institute, Carnegie Mellon University, Pittsburgh, PA 15213 USA (e-mail: camr@ri.cmu.edu; techang@ri.cmu.edu).

P. K. Khosla is with the Department of Electrical Engineering and the Robotics Institute, Carnegie Mellon University, Pittsburgh, PA 15213 USA (e-mail: pkk@ece.cmu.edu).

Digital Object Identifier 10.1109/TRA.2003.817506 branch retinal vein occlusion [4], but which has been found to be generally traumatic when performed with the instrumentation presently available [5]. Additional opportunities for performance improvement exist in otorhinolaryngological, neurological, and microvascular surgery [6].

Various techniques exist for minimization of physiological tremor amplitude during microsurgery. Wrist rests and other supports are common. Many surgeons govern their sleep and their caffeine intake prior to surgery in an attempt to reduce tremor amplitude [7]. Some take beta-blockers, which have been shown to have an attenuating effect [8]. In recent years, attention has turned to the potential of robotic systems to yield much more substantial increases in positioning accuracy. Vitreoretinal microsurgery has been the focus of several efforts, as it is among the most demanding of specialties in terms of manipulation accuracy. There is some amount of consensus among vitreoretinal surgeons on the need for tool tip positioning accuracy approaching $10 \mu \mathrm{m}$ [9], a level that seems to require the assistance of medical robotic devices.

There are several possible robotic approaches to microsurgical accuracy enhancement, each having its advantages and disadvantages. The most common approach is teleoperation [10]-[12]. The physical separation between command input and manipulator output in such systems allows motion scaling and filtering to be applied in a straightforward fashion. Scaled force feedback to the user is likewise facilitated. The drawbacks of such an approach include the high cost of a telerobotic system and the obtrusiveness or unnatural feel of such a system from the point of view of the surgeon, who is accustomed to treating the patient with his own hands. A robotic arm with the requisite workspace for the application also introduces significant safety and liability issues.

To overcome these drawbacks, we have adopted a novel approach: a fully handheld instrument that detects its own motion and deflects its tip for active compensation of the erroneous component of the movement. In the human arm, the surgeon is already in possession of a high degree-of-freedom (DOF) manipulator with high bandwidth, high accuracy (almost, though not quite, adequate for the task), and an unbeatable user interface. Rather than removing this existing manipulator and then working to develop a robotic system that must duplicate many of its features and which faces great disadvantages in terms of naturalness of feel, the philosophy underlying our approach is to retain the advantages possessed by the human surgeon, and to augment only those aspects that require augmentation, such as tip positioning accuracy. Compared to teleoperation, this approach reduces hardware cost by dispensing with the master 
interface and the robotic arm, requiring only a micromanipulator for the instrument tip. The surgeon holds the tool and operates as he has always done, maximizing the transparency or natural feel of the device. The range of the micromanipulator at the instrument tip is designed to be only as much as is needed for compensation of tremor and similar erroneous movements. Safety and liability issues are thus minimized, because even in case of failure, the system is not capable of displacing its tip more than a few hundred microns. If the system is shut down, it simply becomes a passive handheld instrument such as the surgeon has worked with for years. Among the disadvantages of this approach is that in order to perform compensation, the undesired component of movement must be estimated rather than merely suppressed, and any time delay in estimation degrades the quality of the compensation.

Occupying an intermediate position between these two approaches on the continua of both cost and obtrusiveness is the "steady-hand" approach of Taylor et al. [6], in which a robotic arm and the surgeon's hand hold the same instrument simultaneously, the robot sensing the force applied by the human hand and selectively complying in order to suppress unwanted components while allowing the desired movement. As to cost, this approach dispenses with the master interface required for teleoperation, but retains the robotic arm (and thus, many of the concomitant safety and liability issues). As to obtrusiveness, it allows the same immediacy of contact as handheld tools, but does require the user to share the surgical field with a small robotic arm. The telerobotic and steady-hand approaches both allow "third-hand" tasks, in which the surgeon can position a tool and then let go of it, whereas such a thing is impossible with our approach. While force feedback and certain limited kinds of motion scaling are in principle still possible with the steady-hand and handheld compensation approaches, they are certainly more problematic than in the telerobotic scenario.

An approach sharing certain characteristics with both the active handheld and steady-hand approaches was proposed some years ago by Bose et al. [13]. The concept included active error compensation, like our approach (though no estimation algorithm was specified), and a movable base to steady the hand, somewhat resembling the steady-hand approach. However, the proposal seems never to have been fully implemented.

The active handheld instrument presented here, known as "Micron," is designed for vitreoretinal applications, though the principles are of more general applicability. The instrument must perform three functions: motion sensing; filtering (or estimation of erroneous motion); and tip deflection for compensation. The principal areas of research required by the project have been motion quantification and modeling, filtering algorithms, and electromechanical systems for active handheld error compensation. Initial development and evaluation of the tremor filter was reported by Riviere et al. [14]. The present paper reports on the development of a handheld instrument prototype with the necessary sensing and manipulation capabilities to cancel tremor in three dimensions (3-D). It also describes tracking instrumentation that has been developed to serve the needs of the project, and presents preliminary results from evaluation of Micron using this instrumentation.

\section{QUANTIFICATION OF INSTRUMENT MOTION}

Solutions for microsurgical accuracy enhancement are being developed, while in many ways, the problems are not yet fully known. Too little is known about how (and how well) surgeons perform without such enhancement. There has been a need for quantification of tremor amplitude under authentic conditions, and of overall accuracy attainable by the surgeon, in order to establish a performance baseline for enhancement devices. Quantification of the bandwidth utilized by the surgeon, as well as the "performance envelope" in terms of velocity and acceleration, is needed to allow optimal design specification of enhancement devices. Precision measurement systems for microsurgical instruments are also needed in order to enable modeling of tremor and nontremulous error for further development of error estimation algorithms, as well as quantitative testing of devices such as Micron. In response to these needs, we have developed two types of measurement apparatus: an optical instrument tip tracker, designed for performance evaluation of instruments in the laboratory only; and an inertial sensing module, designed for intraoperative sensing of tremor.

\section{A. Laboratory Instrumentation}

Evaluation of microsurgical instruments and development of error estimation algorithms require instrument position tracking in 3-D with accuracy better than $10 \mu \mathrm{m}$. In response to this need, a high-precision 3-D optical tracking system has been developed in our laboratory [15]. Apparatus to Sense Accuracy of Position (ASAP) operates by illuminating the workspace with a high-power infrared LED array (OD-669, Opto Diode Corp., Newbury Park, CA), pulsed at $5 \mathrm{kHz}$, and then using two 2-D position-sensitive detectors (PSDs) (DL-10, UDT Sensor Inc., Hawthorne, CA) to sense the light reflected from a white delrin ball (4.7 $\mathrm{mm}$ diameter) attached to the tip of the surgical instrument. Each PSD is fitted with an absorption filter (over 99\% transmission for wavelengths above $780 \mathrm{~nm}$; less than $0.001 \%$ transmission below $600 \mathrm{~nm})$ and a lens $(10 \mathrm{~mm}$ focal length; with multilayer antireflection coating). The system is shown in Fig. 1.

The converted voltage signals pass through a balanced demodulator (AD630BD, Analog Devices Corp., Norwood, MA). The desired alternating current (ac) component is converted to a direct current $(\mathrm{dc})$ signal, while the dc or low-frequency (erroneous) part is converted to ac. The demodulator output is passed through an active fourth-order Butterworth lowpass filter with a $1200 \mathrm{~Hz}$ cutoff and unity dc gain. Using the detector output signals, the Cartesian coordinates of the centroid of the reflected light spot are computed in analog circuitry. These results are then downsampled from 1500 to $150 \mathrm{~Hz}$. The system is able to track the ball with roughly $1 \mu \mathrm{m}$ root mean square (rms) noise per coordinate [15]. Fig. 2 presents a data sample recorded using ASAP while a subject attempted to hold an instrument motionless. The solid line in the graph shows the subject data; the dotted line shows a sample of the noise for comparison.

Experiments to evaluate overall accuracy and tremor amplitude of trained microsurgeons are ongoing. Initial experiments with three surgeons have yielded an average 3-D vector amplitude of $67 \mu \mathrm{m}$ for physiological tremor in instrument motion, based on recordings of $15 \mathrm{~s}$ duration [16]. ASAP also has potential applications in training of surgeons, including as an input interface to microsurgical simulators. 


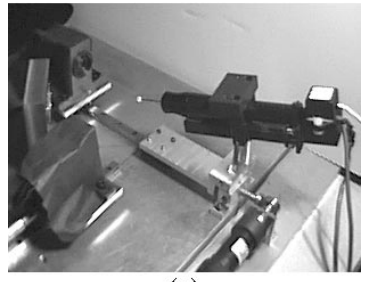

(a)

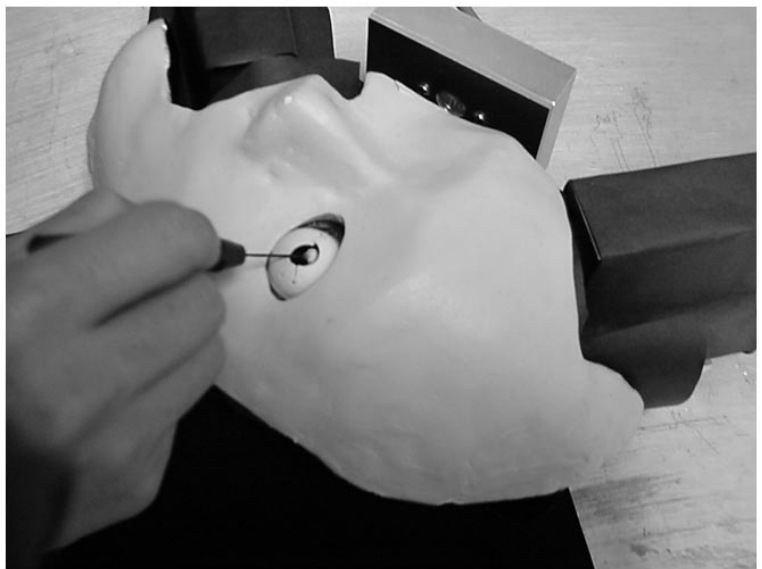

(b)

Fig. 1. ASAP provides tracking of active instruments for evaluation, and allows acquisition of high-precision motion recordings from unassisted surgeons for development of error estimation algorithms. ASAP operates by optically tracking infrared light reflected from the white ball affixed to the tool tip. The figure shows ASAP (a) during testing of Micron (see Section IV-B), and (b) with a face mask used to constrain tool movement for more realistic simulation of vitreoretinal microsurgery.

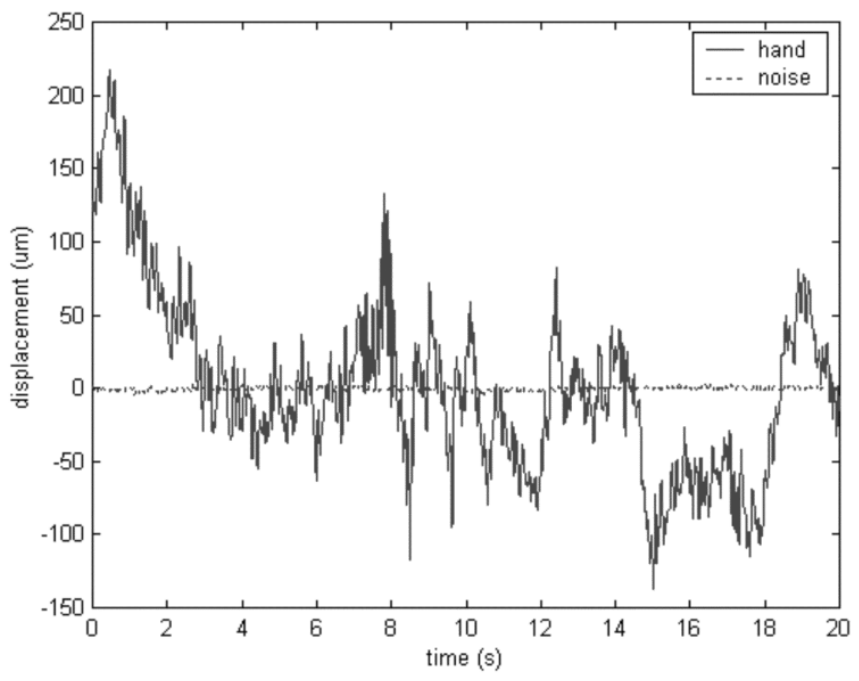

Fig. 2. Sample data recorded by ASAP while a subject attempted to hold an instrument motionless. The dotted line represents a sample of the noise of the system. The graph depicts the vertical $(Z)$ axis of the recording.

\section{B. Intraoperative Instrumentation}

To measure surgical instrument motion under authentic microsurgical conditions, a 6-DOF inertial sensing module has been developed for intraoperative use, containing a CXL02LF3 tri-axial accelerometer (Crossbow Technology, Inc., San Jose, CA) and three CG-16D ceramic rate gyros (Tokin Corp., Tokyo, Japan). The module can be fitted to the proximal end of a microsurgical instrument, and the 3-D velocity of the instrument

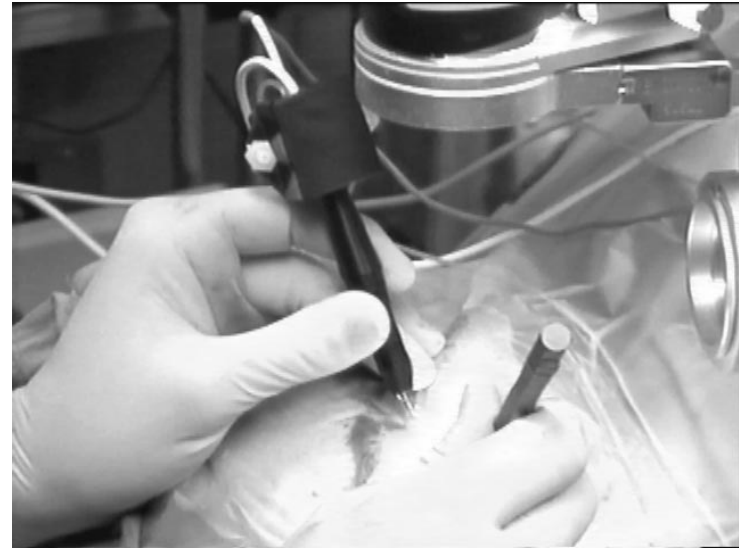

Fig. 3. Recording instrument motion during vitreoretinal microsurgical using inertial sensing. The 6-DOF sensing module, roughly cubical in shape, can be seen at the proximal end of the instrument in the left hand of the surgeon.

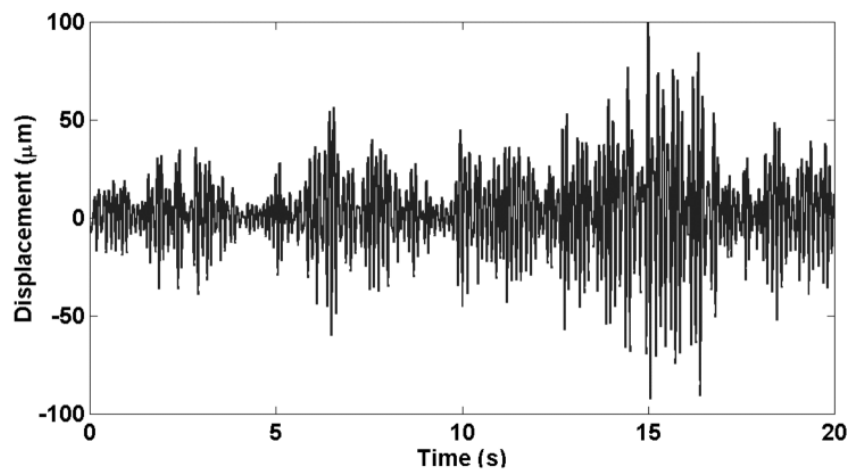

Fig. 4. Sample of physiological tremor recorded during vitreoretinal microsurgery using an inertial sensing module affixed to a surgical instrument. Nominally these data are from a horizontal global coordinate, though this has limited significance due to gyroscope integration drift. The vector sum of rms amplitudes for this tremor data set is $38 \mu \mathrm{m}$.

tip can be obtained via rigid body kinematics. This technique is not intended to track absolute position, because of the inevitable integration drift, but is effective at measuring tremor and other ac components of motion.

This module has been used to record the motion of a DP9603 Madlab Pic-Manipulator (Storz Ophthalmic, Inc., St. Louis, MO) during epiretinal membrane peel procedures, as shown in Fig. 3 [17], [18]. A tremor displacement signal was obtained by integrating the computed tip velocity and then bandpass filtering to the 7-17 $\mathrm{Hz}$ band (these corner frequencies were chosen to provide a flat response in the nominal $8-12 \mathrm{~Hz}$ tremor band). The magnitude of this signal was analyzed for a particular $20 \mathrm{~s}$ segment in which the surgeon was executing the most delicate portion of the membrane removal procedure. The vector sum of the rms amplitudes in the three coordinate directions was $38 \mu \mathrm{m}$. Data from one coordinate direction are shown in Fig. 4.

As for the performance envelope utilized by the surgeon, a raw velocity data set of 5 min duration and an acceleration data set obtained via differentiation of velocity were lowpass filtered with a cutoff frequency of $8 \mathrm{~Hz}$ to remove the physiological tremor and preserve the voluntary components of the motion. Analysis showed that $90 \%$ of the time the velocity was less than $0.34 \mathrm{~m} / \mathrm{s}$, and the acceleration was less than $0.44 \mathrm{~m} / \mathrm{s}^{2}$ [17]. 


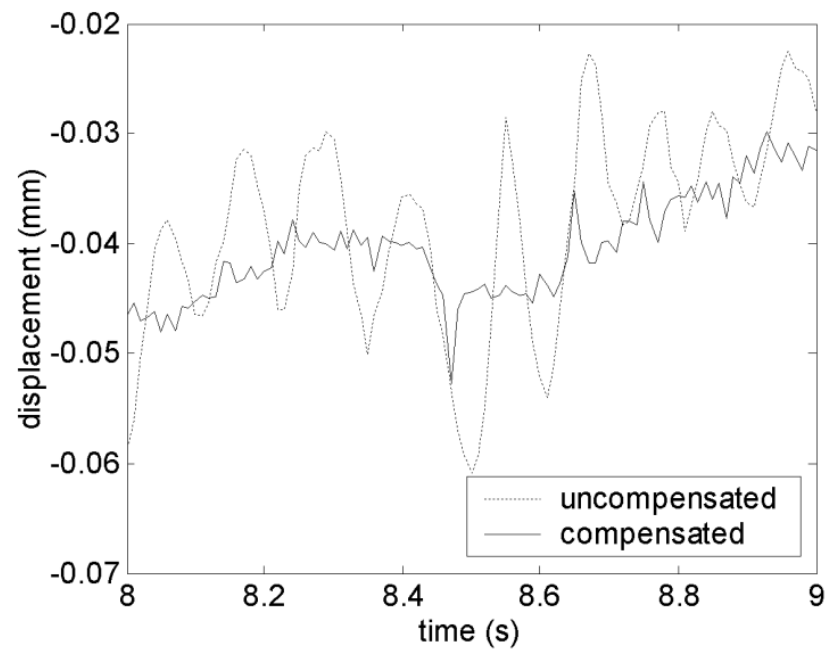

Fig. 5. Sample results from active compensation of recorded physiological tremor in a 1-DOF instrument using tremor estimation via the WFLC algorithm. The hand motion used as input in these experiments was recorded from vitreoretinal surgeons and reproduced using a voice coil actuator. The dashed line represents the reproduced motion as measured at the instrument tip in the absence of compensation. The solid line represents the compensated tip motion for the same input data.

To our knowledge, this represents the first time that instrument movement has been recorded during real microsurgery with sufficient precision to capture physiological hand tremor. These studies are ongoing, with further results to be reported.

\section{FILTERING AlgORITHMS}

\section{A. Tremor}

Tremor is defined as any involuntary, approximately rhythmic, and roughly sinusoidal movement [19]. Therefore, in order to cancel tremor, we have used a system based on the weighted-frequency Fourier linear combiner (WFLC) [14]. The WFLC is a nonlinear adaptive noise canceling algorithm that models the tremor as a sinusoid and tracks its modulation in frequency, phase, and amplitude. In preliminary experiments with a 1-DOF instrument, active canceling of recorded tremor by a system based on the WFLC demonstrated an average rms amplitude reduction of $69 \%$ [14]. Fig. 5 presents typical results from these experiments. The algorithm has also been used for canceling of pathological tremor in assistive computer interfaces [20].

\section{B. Nontremulous Error}

Physiological tremor is the most familiar component of erroneous instrument motion in microsurgery, but there are nontremulous components of erroneous motion, such as myoclonic jerk [9], and these components are often larger than the tremor [21]. Detection and canceling of such nontremulous error is difficult because it may be aperiodic and its frequency content may overlap with voluntary motion. In order to cancel this largely unknown component of erroneous motion, we have begun experiments with a constructive neural network algorithm, the cascade-correlation learning architecture [22], as modified by Nechyba and $\mathrm{Xu}$ for modeling of human control

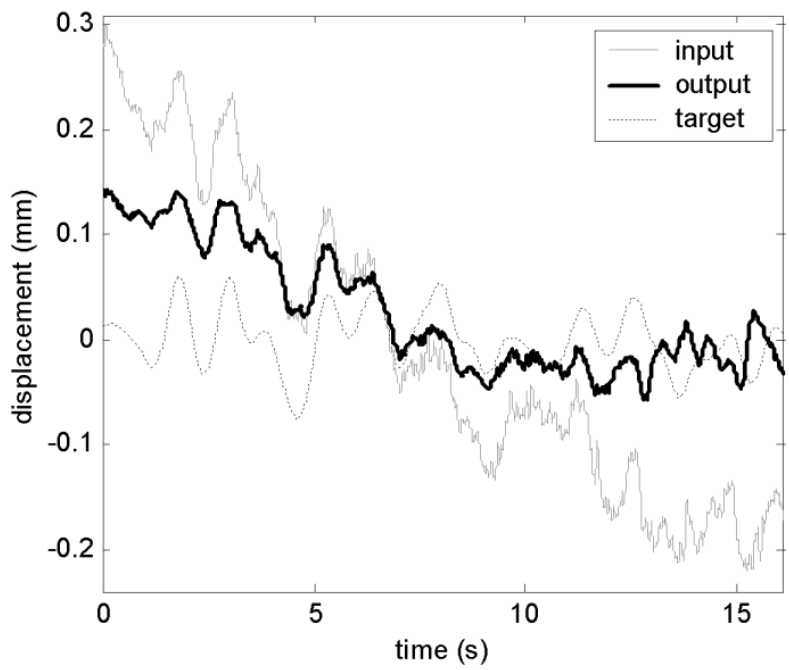

Fig. 6. Typical canceling results for nontremulous error using the cascadecorrelation learning architecture. The target line shows the simulated voluntary motion (white noise bandlimited to $1 \mathrm{~Hz}$ ). The network input for the test is obtained by adding recorded hand motion error to the voluntary motion. The filtered output of the neural network is visibly closer to the target (voluntary) signal than is the original net input. This figure presents testing data only; no network training is taking place in the trial shown.

behavior [23]. This is a versatile technique that enables the neural network to adjust not only the values of its weights, but also the number and transfer functions of its hidden units. The network starts with no hidden nodes, then adds them one by one as error performance stagnates, as determined by a preselected threshold. With each new hidden node, several candidate transfer functions are implemented in parallel, including sigmoids, Bessel functions, and sinusoids, and the one with best performance is selected after a brief period of comparison. Extended Kalman filtering is used for learning, having been found superior in performance to backpropagation [24]. Given an input containing both desired and undesired motion, the network estimates the desired component.

Preliminary tests of this technique in simulation with recorded instrument motion data have yielded an average rms error reduction of $40 \%$ [25]. The signal used in place of voluntary motion in this simulation was an artificial signal consisting of white noise bandlimited to $1 \mathrm{~Hz}$. Fig. 6 shows the results of a typical experiment.

\section{Systems FOR ACTIVE COMPENSATION}

\section{A. Design}

We have developed and built a working prototype of Micron, shown in Fig. 7. This first prototype weighs $170 \mathrm{~g}$, measures $210 \mathrm{~mm}$ in length (including the $30 \mathrm{~mm}$ tool shaft at the tip), and has an average diameter of $22 \mathrm{~mm}$. The narrowed section of the handle near the tip is contoured as an aid to grasping.

Fig. 8 presents a block diagram of the system. Motion sensing is provided by the 6-DOF inertial sensing module described in Section II-B. Tip velocity is calculated and integrated to obtain a displacement signal, from which the erroneous component is estimated using one of the algorithms described in Section III. The estimated displacement error is then used as an input command to the manipulator, driving the tip to generate an equal but 


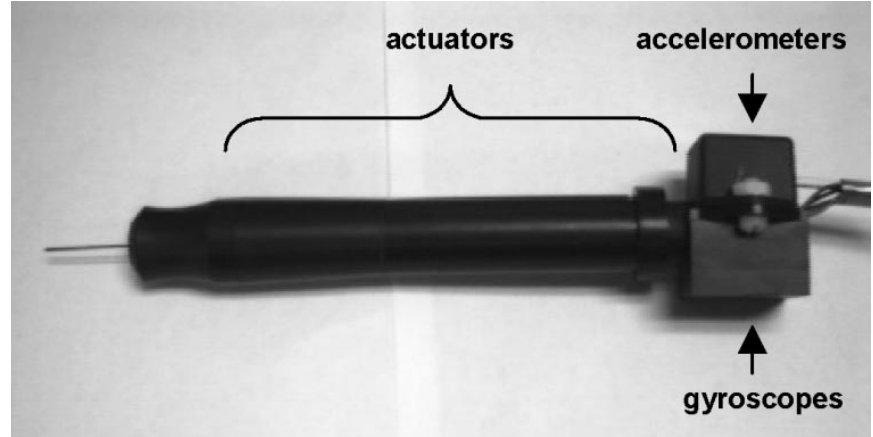

Fig. 7. Micron, a handheld microsurgical instrument for active compensation of tremor and other movement errors.

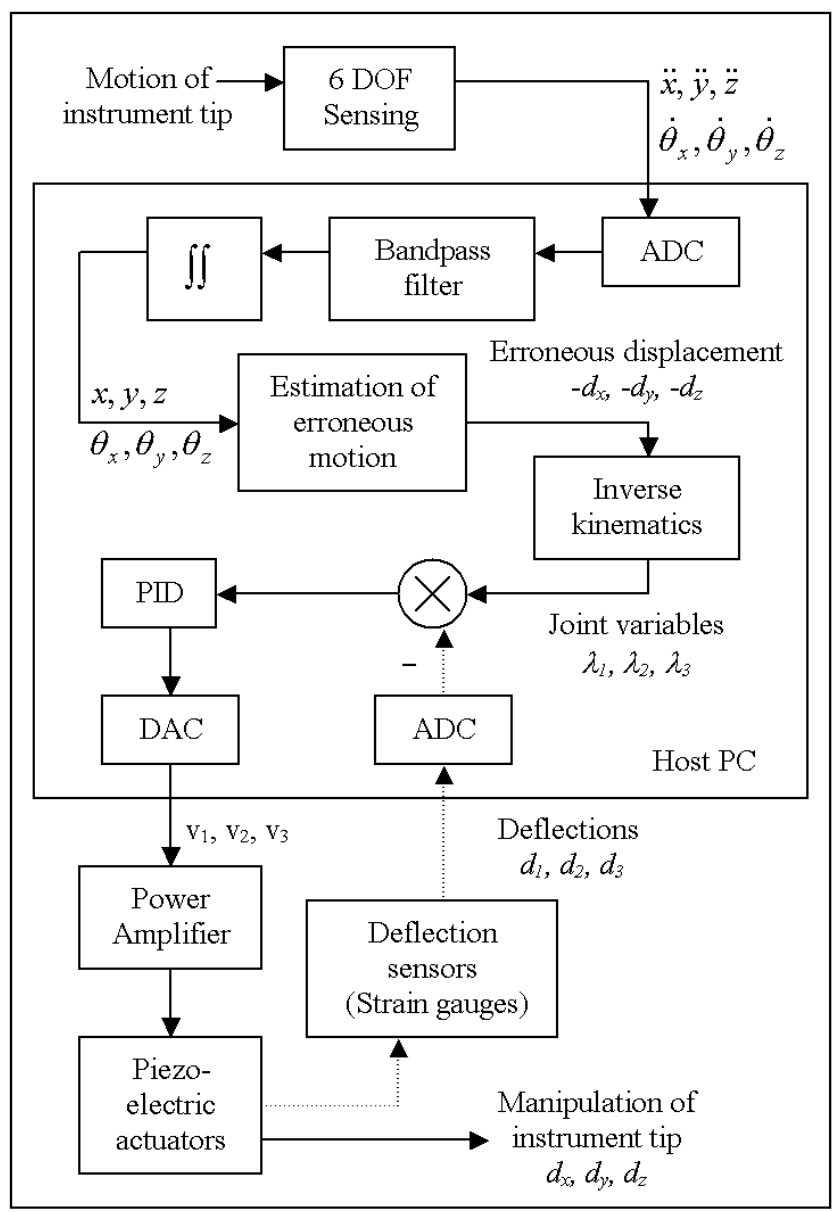

Fig. 8. Block diagram of Micron. Closed-loop control (dotted line and "PID" block) will be implemented in the second-generation prototype.

opposite deflection to compensate the erroneous motion. Position control of the manipulator was chosen in order to facilitate keeping the motion of the shaft centered about the null point of the manipulator, avoiding the limits of the workspace.

The present system controls the piezoelectric actuators in an open loop; there are no sensors to measure the displacement of the manipulator. Work is presently underway to incorporate strain gauges for this purpose in future prototypes of Micron, thus closing the control loop, as depicted by the dotted line in Fig. 8. (The adaptive noise canceler does close a certain loop, but

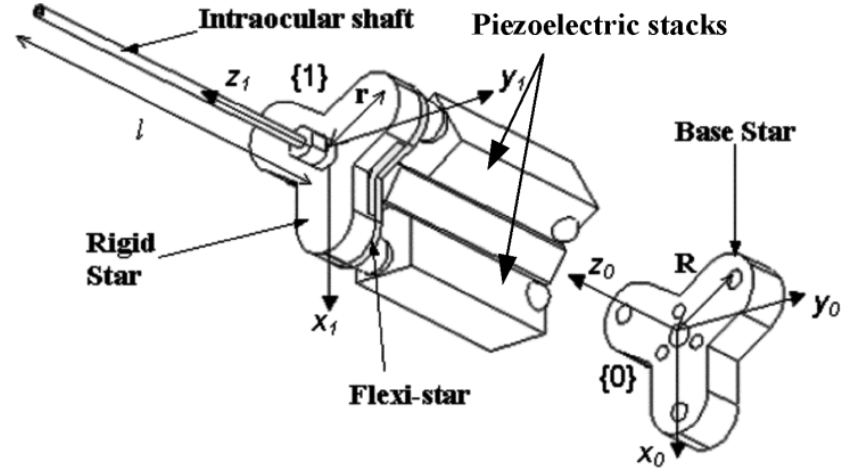

Fig. 9. The manipulator at the instrument tip. The plastic "flexi-star" constrains the manipulator against rotation about the long axis and translation in directions transverse to this axis. Its center is clamped to the stationary center column of the instrument, and the tip of each leg is pinned to the corresponding leg of the rigid star which holds the intraocular tool shaft. Each actuator consists of seven piezoelectric stacks, with the last stack resting against the base star. Only the first stack of each actuator is shown.

this is solely in software, within the "Estimation of erroneous motion" block in Fig. 8.)

Estimation of erroneous motion is performed at $1 \mathrm{kHz}$ sampling using the filtering approach described in [14], with the following parameter values: $\mu=0.25, \mu_{0}=10^{-7}, \mu_{b}=0.005$, $\hat{\mu}=0.3, M=1, \mathbf{w}_{0}=\mathbf{0}$, and $\omega_{0_{0}}=0.0628(10 \mathrm{~Hz})$.

The workspace is small enough that changes in shaft orientation due to the micromanipulator deflection are negligible with regard to the performance of practical surgical tasks. This reduces the dimension of the configuration space of the manipulator to three, and simplifies the mechanical design and the online computation of inverse kinematics. A parallel manipulator design is best suited to this application because of its rigidity, compactness, and simplicity in design, as compared with a serial mechanism.

Piezoelectric actuators were chosen for their high bandwidth. The TS18-H5-202 piezoelectric stack actuator (Piezo Systems, Inc., Cambridge, MA) measures $5 \mathrm{~mm} \times 5 \mathrm{~mm} \times 18 \mathrm{~mm}$, and deflects to a maximum of about $14.5 \mu \mathrm{m}$ with an applied voltage of $100 \mathrm{~V}$. It offers relatively good control linearity, response time of $50 \mu \mathrm{s}$, and actuation force of up to $840 \mathrm{~N}$. A range of motion of $100 \mu \mathrm{m}$ or greater has been achieved in each of the three coordinate directions by stacking seven piezoelectric elements to form each actuator.

Fig. 9 depicts the robotic manipulator that deflects the tip of the instrument. It is a 3-DOF parallel design with kinematics similar to [26]. The stainless steel tool shaft is affixed to the center of a three-pointed rigid star. Beneath the rigid star is a thin flexible star made of ABS 780 thermoplastic. The tip of each leg of the rigid star is pinned to the tip of the corresponding leg of the flexible star. At its center, the flexible star is bolted to the triangular center column, constraining it against axial rotation and against translation in the two coordinates transverse to the long axis of the instrument. The maximum attainable tip displacement and velocity of the manipulator are summarized in Table I. Experimental results show that the prototype is able to track 1-D and 3-D trajectories with rms error of $2.5 \mu \mathrm{m}$ and 11.2 $\mu \mathrm{m}$, respectively [27]. 
TABLE I

MAXIMUM DiSPLACEMENT AND VELOCITY OF MiCROMANIPULATOR

\begin{tabular}{ccc}
\hline \hline & Transverse $(\mathrm{X} \& \mathrm{Y})$ & Axial $(\mathrm{Z})$ \\
\hline Max. tip displacement $(\mu \mathrm{m})$ & 560 & 100 \\
Max. tip velocity $(\mathrm{m} / \mathrm{s})$ & 11.2 & 2 \\
\hline \hline
\end{tabular}

TABLE II

Average ERror Reduction Performance of Micron in Each INSTRUMENT AXIS OVER 12 TRIALS

\begin{tabular}{c|ccc}
\hline \hline Axis & $\begin{array}{c}\text { Uncompensated } \\
\text { Amplitude }(\mu \mathrm{m} \text { p-p })\end{array}$ & $\begin{array}{c}\text { Compensated } \\
\text { Amplitude }(\mu \mathrm{m} \text { p-p })\end{array}$ & $\begin{array}{c}\text { Reduction } \\
(\%)\end{array}$ \\
\hline $\mathrm{X}$ & 39 & 31 & 21 \\
$\mathrm{Y}$ & 43 & 37 & 14 \\
$\mathrm{Z}$ & 70 & 34 & 51 \\
\hline \hline
\end{tabular}

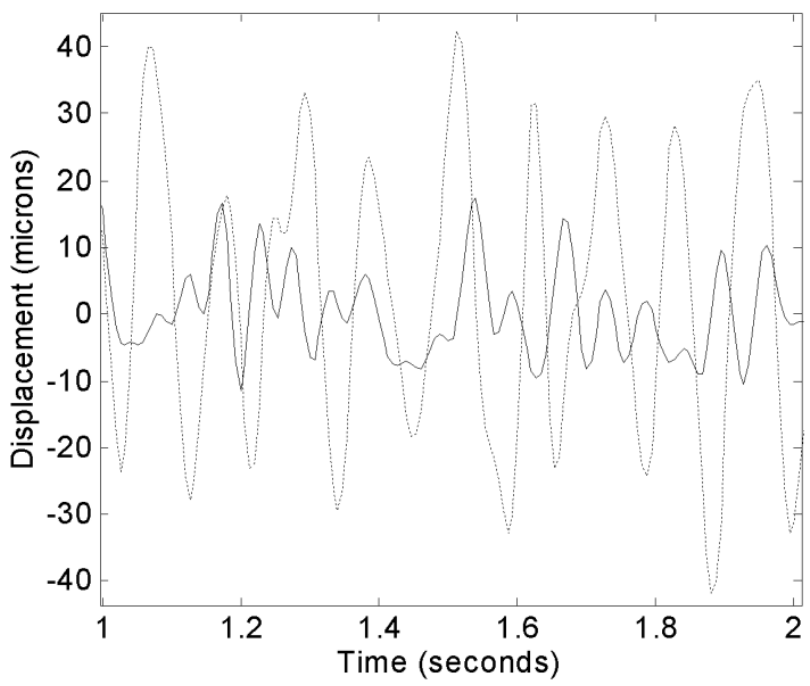

Fig. 10. Performance of Micron in 1-D noise canceling test, with motion parallel to the long axis of the instrument. The dotted line depicts the uncompensated motion and the solid line the compensated motion.

\section{B. Experimental Methods}

Preliminary testing of Micron with WFLC-based tremor canceling has been conducted using a motorized testbed, shown in Fig. 1(a), to generate oscillatory motion, simulating the hand tremor of the surgeon [28]. The oscillating plate on which Micron was mounted rested on a linear slide. A spring-loaded driving shaft, attached to the back end of the plate, was driven at roughly $9 \mathrm{~Hz}$ by a cam rotated using a de servomotor.

Two types of tests were conducted.

- 1-D, involving motion only along the long axis $(Z)$ of the instrument, at $50 \mu \mathrm{m}$ peak-to-peak (p-p).

- 3-D, using a $90 \mu \mathrm{m}$ p-p oscillation. Table II shows the uncompensated magnitude of input oscillation in each of the three body coordinates of the instrument.

In each case, 12 trials were conducted. A small marker ball was mounted at the instrument tip, and ASAP was used to track tip position. The instrument sampled data at $1000 \mathrm{~Hz}$.

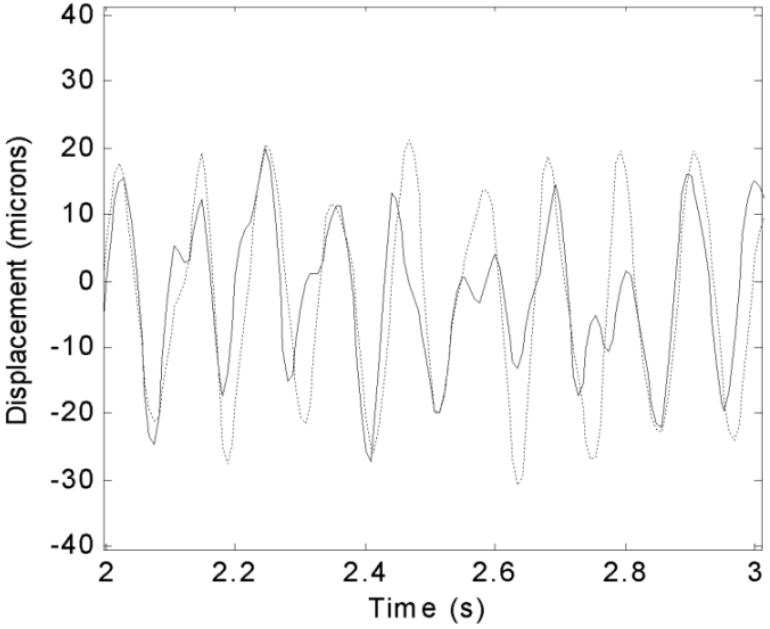

(a)

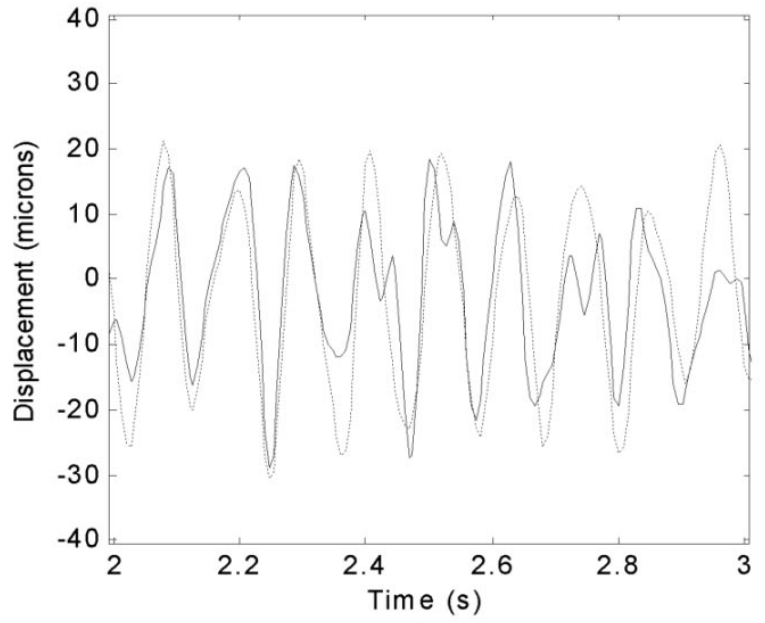

(b)

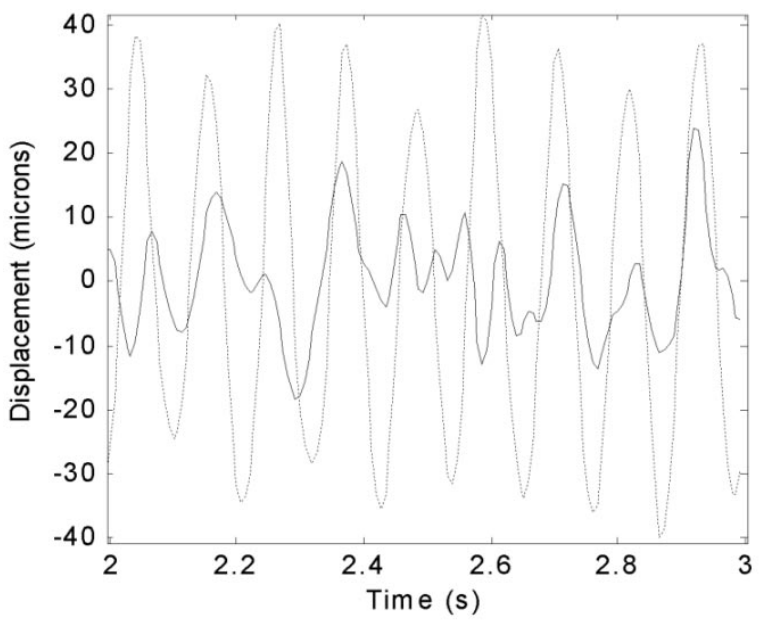

(c)

Fig. 11. Performance of instrument in 3-D noise canceling test. The dotted line depicts the uncompensated motion and the solid line the compensated motion. (a) $X$ axis (transverse to the long axis of the instrument). (b) $Y$ axis (transverse). (c) $Z$ axis (axial).

Rather than report rms values, we have multiplied rms results by $2 \sqrt{ } 2$ to obtain the equivalent $\mathrm{p}-\mathrm{p}$ amplitude that would result if the signal were a perfect sinusoid. This is proposed as a more clinically meaningful metric, as the surgeon is interested in the overall excursion of undesired motion, not in its rms amplitude. 


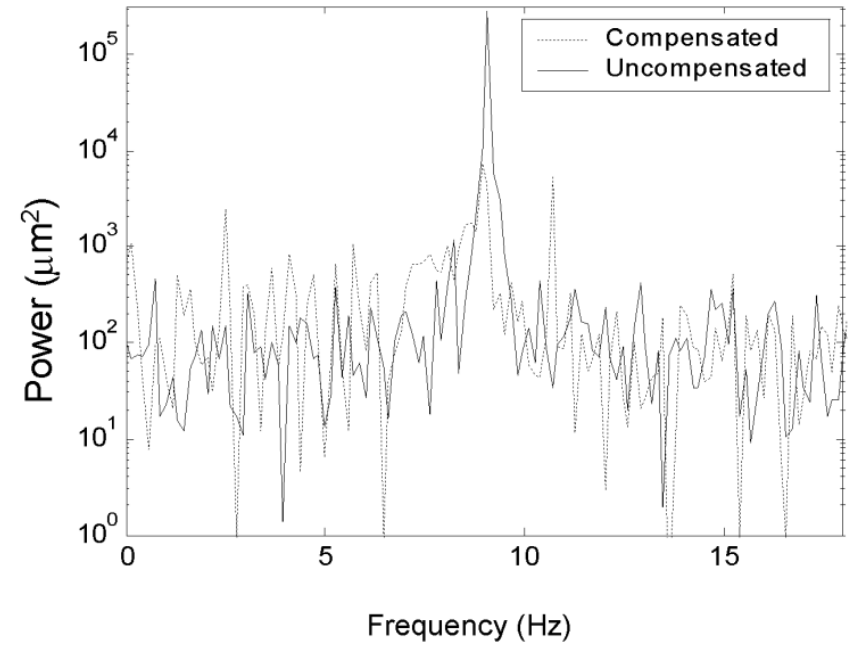

Fig. 12. Amplitude spectral density from a 3-D noise canceling test, comparing the tip motion with and without compensation.

\section{Experimental Results}

In the tests involving only axial motion, the average erroneous motion amplitude was reduced from $51 \mu \mathrm{m}$ p-p to $25 \mu \mathrm{m}$ p-p, a reduction of $51 \%$. Sample results from the 1-D tests are shown in Fig. 10.

In 3-D canceling tests, the average vector amplitude of erroneous motion was reduced from $91 \mu \mathrm{m}$ p-p to $60 \mu \mathrm{m} \mathrm{p}-\mathrm{p}$, representing a $34 \%$ reduction over 12 trials. Table II shows the average equivalent $\mathrm{p}-\mathrm{p}$ amplitude and the average error reduction for each of the three axes. Fig. 11 shows typical results for the 3-D tests. The spectral content of a typical 3-D test can be seen in Fig. 12.

\section{DISCUSSION}

The results presented demonstrate the feasibility of the general approach of active 3-D tremor compensation in a handheld microsurgical instrument using inertial sensing, piezoelectric actuation, and adaptive tremor canceling based on the WFLC algorithm. The accuracy of the present instrument prototype is limited to a noise level of roughly $30 \mu \mathrm{m}$ p-p, largely due to sensing inaccuracies. This effect can be seen in Fig. 11, in that the initial error is considerably larger in $z$ than in $x$ and $y$, but all three are reduced to roughly $30 \mu \mathrm{m}$ p-p by the active canceling. In other words, performance in $x$ and $y$ seems worse than in $z$ only because the input signal is smaller. The effect is also evident in Fig. 12, in which the main peak at $9 \mathrm{~Hz}$ has been suppressed, but increased noise is noticeable at other frequencies. The manipulator has demonstrated higher accuracy [27], as has the WFLC in 1-DOF tremor canceling tests [14]. It is expected that improved sensor signal filtering and closed-loop control will improve performance. Development of a second-generation prototype incorporating these features is underway.

The first prototype was developed as a proof of concept; its size and weight are near the limits of feasibility. The second prototype is smaller (130 mm length, $19 \mathrm{~mm}$ diameter) and lighter (100 g). Plans for the new prototype include experiments with recorded hand tremor, followed by live tests performed by trained surgeons and also by cell biologists, for whom Micron has clear relevance as a handheld micromanipulator for laboratory use. In addition, investigation of neural network-based methods such as those in [25] for canceling of nontremulous error continues, to be followed by implementation in Micron. Additional surgical applications are also being explored, including handheld laser microsurgery in a variety of clinical specialties.

The applications of the handheld robotic device that we have developed do not end at compensation of involuntary hand motion. We expect it to serve as a platform for the development of more intelligent instruments that can adapt to specific users and procedures, tune their parameters for optimal performance, and implement macros to partially automate repetitive or stereotyped surgical tasks.

\section{CONCLUSION}

A handheld microsurgical instrument that performs active compensation of erroneous motion has been developed and is undergoing testing and refinement.

\section{ACKNOWLEDGMENT}

The authors are grateful to L. Hotraphinyo and S. P. N. Singh for research assistance, and to Drs. R. Hollis and A. Rizzi for the use of circuit hardware for ASAP.

\section{REFERENCES}

[1] M. Patkin, "Ergonomics applied to the practice of microsurgery," Aust. N. Z. J. Surg., vol. 47, pp. 320-239, 1977.

[2] P. Mitchell, W. Smith, and A. Chang, "Prevalence and associations of retinal vein occlusion in Australia: The blue mountains eye study," Arch. Ophthalmol., vol. 114, pp. 1243-1247, 1996.

[3] J. N. Weiss, "Treatment of central retinal vein occlusion by injection of tissue plasminogen activator into a retinal vein," Amer. J. Ophthalmol., vol. 126, pp. 142-144, 1998

[4] E. M. Opremcak and R. A. Bruce, "Surgical decompression of branch retinal vein occlusion via arteriovenous crossing sheathotomy," Retina, vol. 19, pp. 1-5, 1999.

[5] W. N. Tang and D. P. Han, "A study of surgical approaches to retinal vascular occlusions," Arch. Ophthalmol., vol. 118, pp. 138-143, 2000.

[6] R. Taylor, P. Jensen, L. Whitcomb, A. Barnes, R. Kumar, D. Stoianovici, P. Gupta, Z. Wang, E. de Juan, Jr., and L. Kavoussi, "A steady-hand robotic system for microsurgical augmentation," Int. J. Robot. Res., vol 18, pp. 1201-1210, 1999.

[7] M. U. Humayun, R. S. Rader, D. J. Pieramici, C. C. Awh, and E. de Juan, Jr., "Quantitative measurement of the effects of caffeine and propranolol on surgeon hand tremor," Arch. Ophthalmol., vol. 115, pp. 371-374, Mar. 1997.

[8] M. J. Elman, J. Sugar, R. Fiscella, T. A. Deutsch, J. Noth, M. Nyberg, K. Packo, and R. J. Anderson, "The effect of propranolol versus placebo on resident surgical performance," Trans. Amer. Ophthalmol. Soc., vol. 96, pp. 283-291, 1998.

[9] S. Charles, "Dexterity enhancement for surgery," in Computer Integrated Surgery: Technology and Clinical Applications, R. H. Taylor, S. Lavallée, G. C. Burdea, and R. Mösges, Eds. Cambridge, MA: MIT Press, 1996, pp. 467-471.

[10] H. Das, H. Zak, J. Johnson, J. Crouch, and D. Frambach, "Evaluation of a telerobotic system to assist surgeons in microsurgery," Comput.-Aided Surg., vol. 4, pp. 15-25, 1999.

[11] P. S. Jensen, K. W. Grace, R. Attariwala, J. E. Colgate, and M. R. Glucksberg, "Toward robot-assisted vascular microsurgery in the retina," Graefes Arch. Clin. Exp. Ophthalmol., vol. 235, pp. 696-701, Nov. 1997. 
[12] I. W. Hunter, T. D. Doukoglou, S. R. Lafontaine, P. G. Charette, L. A. Jones, M. A. Sagar, G. D. Mallinson, and P. J. Hunter, "A teleoperated microsurgical robot and associated virtual environment for eye surgery," Presence, vol. 2, pp. 265-280, 1993.

[13] B. Bose, A. K. Kalra, S. Thukral, A. Sood, S. K. Guha, and S. Anand, "Tremor compensation for robotics assisted microsurgery," in Proc. 13th Annu. Int. Conf. IEEE Biomedical Engineering Society, vol. 3, Paris, France, 1992, pp. 1067-1068.

[14] C. N. Riviere, R. S. Rader, and N. V. Thakor, "Adaptive canceling of physiological tremor for improved precision in microsurgery," IEEE Trans. Biomed. Eng., vol. 45, pp. 839-846, July 1998.

[15] L. Hotraphinyo and C. N. Riviere, "Precision measurement for microsurgical instrument evaluation," in Proc. 22nd Annu. Int. Conf. IEEE Engineering in Medicine and Biology Society, Istanbul, Turkey, 2001, pp. 3454-3457.

[16] - "Three-dimensional accuracy assessment of eye surgeons," in Proc. 22nd Annu. Intl. Conf. IEEE Engineering in Medicine and Biology Society, Istanbul, Turkey, 2001, pp. 3458-3461.

[17] S. P. N. Singh and C. N. Riviere, "Physiological tremor amplitude during retinal microsurgery," in Proc. IEEE 28th Annu. Northeast Bioengineering Conf., 2002, pp. 171-172.

[18] C. Riviere and P. S. Jensen, "A study of instrument motion in retinal microsurgery," in Proc. 21st Annu. Int. Conf. IEEE Engineering in Medicine and Biology Society, Chicago, IL, 2000, pp. 59-60.

[19] R. J. Elble and W. C. Koller, Tremor. Baltimore, MD: Johns Hopkins Univ. Press, 1990, p. 1.

[20] C. N. Riviere and N. V. Thakor, "Modeling and canceling tremor in human-machine interfaces," IEEE Eng. Med. Biol. Mag., vol. 15, pp. 29-36, May/June 1996.

[21] C. N. Riviere, R. S. Rader, and P. K. Khosla, "Characteristics of hand motion of eye surgeons," in Proc. 19th Annu. Int. Conf. IEEE Engineering in Medicine and Biology Society, Chicago, IL, 1997, pp. $1690-1693$

[22] S. E. Fahlman and C. Lebiere, "The cascade-correlation learning algorithm," in Advances in Neural Information Processing Systems 2, D. S. Touretzky, Ed. San Mateo, CA: Morgan Kaufman, 1990, pp. 524-532.

[23] M. C. Nechyba and Y. Xu, "Learning and transfer of human real-time control strategies," J. Adv. Computat. Intell., vol. 1, no. 2, pp. 137-154, 1997.

[24] G. V. Puskorius and L. A. Feldkamp, "Decoupled extended Kalman filter training of feedforward layered networks," in Proc. Int. Joint Conf. Neural Networks, vol. 1, 1991, pp. 771-777.

[25] W. T. Ang and C. N. Riviere, "Neural network methods for error canceling in human-machine manipulation," in Proc. 22nd Annu. Int. Conf. IEEE Engineering in Medicine and Biology Society, Istanbul, Turkey, 2001, pp. 3462-3465.

[26] K.-M. Lee and D. K. Shah, "Kinematic analysis of a three-degrees-of-freedom in-parallel actuated manipulator," IEEE Trans. Robot. Automat., vol. 4, pp. 354-360, Apr. 1988.

[27] W. T. Ang, C. N. Riviere, and P. K. Khosla, "An active hand-held instrument for enhanced microsurgical accuracy," in Proc. Medical Image Computing and Computer-Assisted Intervention-MICCAI 2000, vol. 1935, 2000, pp. 878-886.

[28] — - "Design and implementation of an active hand-held instrument for enhanced microsurgical accuracy," in Proc. IEEE/RSJ Joint Conf. Intelligent Robots and Systems, Maui, HI, 2001, pp. 1106-1111.

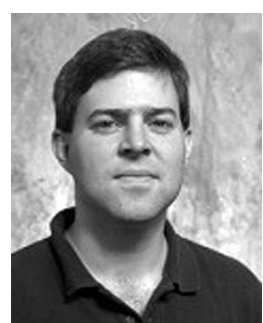

Cameron N. Riviere (S'94-M'96) received B.S. degrees in aerospace engineering and ocean engineering from the Virginia Polytechnic Institute and State University, Blacksburg, in 1989, and the Ph.D. degree in mechanical engineering from The Johns Hopkins University, Baltimore, MD, in 1995.

Since 1995, he has been with the Robotics Institute at Carnegie Mellon University, Pittsburgh, PA, where he is presently a Systems Scientist. His research interests include medical robotics, control systems, signal processing, learning algorithms, and biomedical applications of human-machine interfaces.

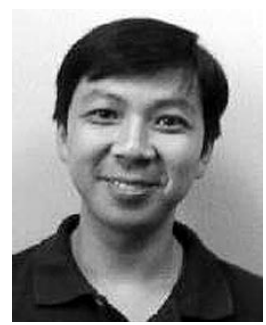

Wei Tech Ang (S'98) received the B.Eng. and M.Eng. degrees in mechanical and production engineering from Nanyang Technological University, Singapore, in 1997 and 1999, respectively. He is currently working toward the Ph.D. degree in the Robotics Institute at Carnegie Mellon University, Pittsburgh, PA.

His research interests include medical robotics, mechatronics, mechanism design, kinematics, signal processing, and learning algorithms.

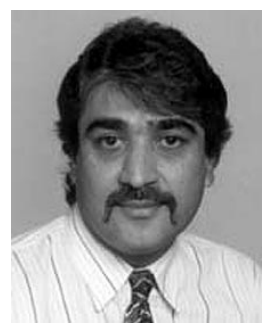

Pradeep K. Khosla (F'95) received the B. Tech. degree (Honors) from the India Institute of Technology, Kharagpur, India, and the M.S. degree in 1984 and the Ph.D. degree in 1986 from Carnegie Mellon University, Pittsburgh, PA.

At Carnegie Mellon University, he served as Assistant Professor of ECE and Robotics from 1986 to 1990, Associate Professor from 1990 to 1994, Professor since 1994, and Founding Director of the Institute for Complex Engineering from 1997 to 1999 . He is currently the Philip and Marsha Dowd Professor of Engineering and Robotics and Head of the Electrical and Computer Engineering Department. From January 1994 to August 1996, he served as a DARPA Program Manager. His research interests are in the areas of Internet-enabled collaborative design and distributed manufacturing, agent-based architectures for distributed design and embedded control, software composition and reconfigurable software for real-time embedded systems, and reconfigurable and distributed robotic systems. His research has resulted in two books and more than 200 journal articles, conference papers, and book contributions. He is currently an Associate Editor for the ASME Journal of Computers and Information Science in Engineering (JCISE).

Dr. Khosla received the Carnegie Institute of Technology Ladd award for excellence in research in 1989, the ASEE 1999 George Westinghouse Award for Education, the Siliconindia Leadership award for Excellence in Academics and Technology in 2000, and the W. Wallace McDowell award from the IEEE Computer Society in 2001. He was appointed a Distinguished Lecturer for the IEEE Robotics and Automation Society for 1998-2001. He was the General Chairman for the 1990 IEEE International Conference on Systems Engineering, Program Vice Chairman of the 1993 International Conference on Robotics and Automation, General Co-Chairman of the 1995 Intelligent Robotics Systems (IROS) conference, and Program Vice-Chair for the 1997 IEEE Robotics and Automation Conference. He has served as Technical Editor of the IEEE TRANSACTIONS ON ROBOTICS AND AUTOMATION. 